\title{
Index rerum ad Vol. 21
}

\section{Confecit Wilhelm Baumann, Zurich}

Aguas minerales, 193

Angioma renis, 18

Anuria, prevention with Mannitol, 54

Bassinet, operations des tumeurs, 520 Bladder, complications after X-radia-

tion, 393, 437 -, complications following hysterectomy,

444,452 -, experimental studies, 182 -, resorption of radioactive-tagged

substances, 538

Carcinoma prostatae, 77 Cystinurie, 477 Cysto-plasties, 477

Dimethylnitrosamine, renal carcino-genesis, 229

Eaux minerales, 193

Epididymis, granulomatous lesioi > s, 564

Estrechez uretral post-traumatica, 209

Fertilitätsstörungen des Mannes, Immunreaktionen, 247

Fertility disturbances, immune reactions, 247

Fibrinolyseblutung, Behandlung mit p-Aminomethylbenzoesäure, 61

Gemmangiom, 18

Granulome du testicule et de Гépidi-dyme, 564

Gynecological surgery, ureteral complications, 452

- -, vesical complications, 444, 452

Haemangioblastom, 18 Haemangiosarkom, 18 Haematurie bei Angiom der Niere, 18

Harnröhrenstriktur, posttraumatische,

209 Harnblase, Experimente über Öffnungs-

und Schließungsmechanismus, 182 -, Komplikationen nach Bestrahlungen,

393, 437

-, Komplikationen nach Hysterektomie,

444 -, Resorption radioaktiver Substanzen,

538 Harnreflux, 531 Harnröhrenstenosen, Resultate der

Johanson-Plastik, 465 Hidronefrosis producida experimental-

mente, 1 Histokompatibilitätsmessung, 173 Hoden, granulomatöse Veränderungen,

564 Hypernephroma, 365 Hydronephrosis experimentalis, 1 Hysterectomy, vesical

complications,

444,452

Immune reactions in male fertility disturbances, 247

Immunreaktionen bei Fertilitätsstörungen des Mannes, 247

Impotentia generandi posttraumatica, 239

Infusionsurographie, 489

Intravenous urography, complications, 500

Irradiation of the kidney, renal changes, 511 
Kidney, carcinogenesis, 229

-, multiple bilateral angioma, 18

-, the action of oestrogens, 47

Lavage pyélique, 36 Lithiase urinaire, 477

Mannitol, treatment of patients with

uremia, 28, 54, 375, 381 Mineral waters, 193

Nebenhoden, granulomatöse Veränderungen, 564

Nephrolithiasis, 477

Niere, Carcinogenese, 229

-, Einfluß der Oestrogene auf die Rinde, 47

-, Haematurie bei Angiom, 18

Index

rerum

579

-, multiple doppelseitige Angiome, 18 Nierenbecken, organerhaltende Opera-

tionen, 520 Nierenbeckenspülungen nach Timmer-

mann, 36 Nierentransplantation, 97-181, 386 -, operative Technik, 124 -, Spenderwahl, 120 -von

Leichennieren, 131 Nierentuberkulose, Behandlung mit Cor-

ticoiden, 307 Nierenveränderungen nach Betrahlung

der Niere, 511

Oestrogenwirkung auf die Nierenrinde,

47 Oxalurolithiasis, 338

Para-aminomethylbenzoic acid, treatment of post-prostatectomy bleeding, 61

Pelvis renal, operaciones de los tumores, 520

Plastic operations, 477

Prostata, ACTH-Belastung und Leber-schädigung, 553

Prostata, Overloading of AGTH and liver damage, 553

Prostatectomia supra pubica, 549

Prostatectomy, persistence of prostatic bed, 68

Prostatectomy, treatment of bleeding with PAMBA, 61

Prostatic carcinoma, 77

- $\quad$ tuberculosis, 329

Pseudopapillomata, spinal cord injuries,

353

Reflux urinaire, 531

Rein, action des æstrogènes, 47

-, angiomes multiples bilatéraux, 18

-, etude carcinogénétique, 229

-, lavage pyélique, 36

-, transplantation, 97-181, 386

Renal carcinogenesis, 229

changes after irradiation of the kidney, 511

pelvic lavage, 36

tumors, surgery, 520

- $\quad$ transplantation, 97-181, 386 
of cadaver kidneys, 131

, operative technic, 124

, selection of donor, 120

coids, 307

tuberculosis, treatment with corti-

Renovasographie bei Angiom der Niere,

18 Rétrécissement uréthral posttraumati-

que, 209 Riñon, acción de los estrogenos, 47 -, angiomas bilaterales, 18 -, carcinogenesis, 229 -, lavado piélico, 36 -, trasplante, 97-181, 186 Röntgenbestrahlungen, Blasenkompli-

kationen, 393, 437 Rückenmarksverletzungen, Pseudo-

papillome, 353

Spinal cord injuries, pseudopapillomata,

353 Strahlencystitis, 393, 437 Suprapubic prostatectomy, 549

Testicle, granulomatous lesions, 564 Timmermann, Nierenbeckenspülungen,

36 Transplantation du rein de cadavre, 131

- $\quad$ rénale, éthique médicale, 141

, choix du greffon, 120

, technique operatoire, 124

Trasplante de riñón de cadaver, 131

- $\quad$ renal, 97-181, 386

, elección del injerto, 120

, técnica operatoria, 124

Tuberculose de la prostate, 329

urinaire, traitement avec des corti-coïdes, 307

uro-génitale, modifications hormona-les, 289

Uremia, treatment with Mannitol, 28, 54, 375, 381

Ureteral complications following gynecological surgery, 452

Uréthral stenosis, Johanson plastic operation, 465

- $\quad$ stricture, posttraumatic, 209

Uréthro-plasties, 477

Uréthro-plasties selon Johanson, résul-

tats, 465

Urinary reflux, 531

Urogenital tuberculosis, hormonal variations, 289

580

Index

rerum

Urografia por perfusion, 489 Urographie intraveineuse, complications, 500 Urolithiasis, 338, 477

Vejiga, complicaciones después de la

irradiación, 393, 437 -, complicaciones después histerectomia,

444, 452 -, estudio experimental, 182 Vessie, complications après hysterecto-

mie, 444,452

-j complications après irradiation, 393 ,

437 -, etude expérimentale, 182 -j rêsorption de substances radioactives,

538

X-Radiation, renal complications, 511 X-Radiation, vesical complications, 393, 
